\title{
RM05
}

\section{Topology vs. Topography: Sometimes Less is} More

B. Thomson* (ExxonMobil Production Co) 


\section{EAGE}

\section{Introduction}

The software tools we use for interpretation and modelling are powerful and complex. The outputs they generate are always extremely precise (to the $\mathrm{n}^{\text {th }}$ decimal place!) but may not always be as reliable as we wish them to be. To support business decisions we required integrated work products that are reliable rather than being merely precise. So how do we move from precision to reliability?

\section{Topology vs Topography}

In the context of reservoir modelling, Topology may be considered a representation of the reservoir and fluid system as a series of nodes (compartments and wells) and connections (flow units and their boundaries). In contrast; Topography is the representation of the reservoir system as a series of maps and models that contain a lot of detail and precision. A simple example which illustrates the difference between Topology and Topography is the London Tube Map.

Topology of Tube connections

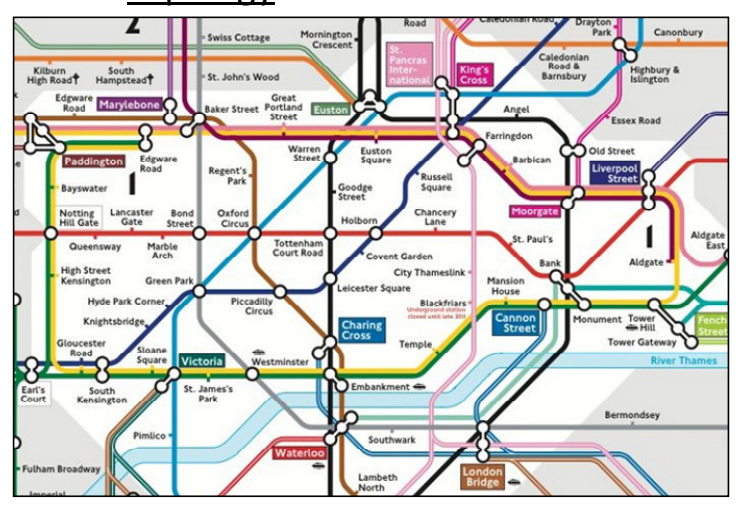

Topography of Tube connections

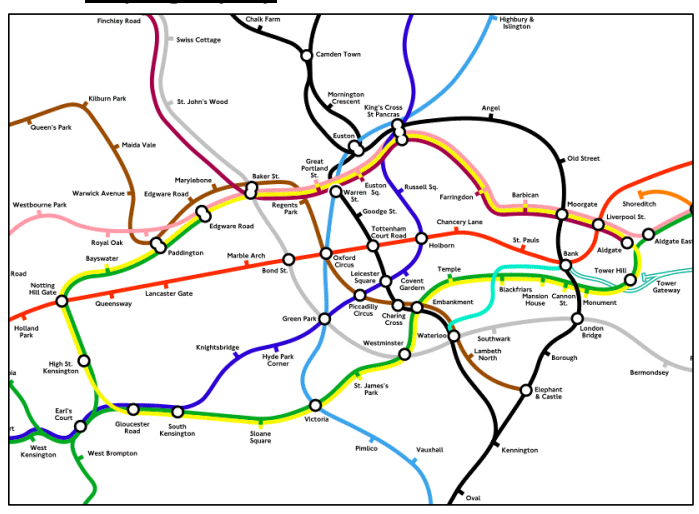

In this example the topological representation is not precise but it is reliable and contains all the key information we require. The addition of the topographic detail adds precision but not a lot of utility.

Building complex and detailed reservoir models that contain great precision (topography) can occur without having articulated and defined the underlying topology we are trying to represent. The use of schematic cross sections, cartoons, and structure maps with fluid distributions all facilitate a shared understanding of the topology being represented with the model. The use of these simple work products often helps break down communication issues between different disciplines.

\section{Application to uncertainty}

If we create a simple representation of the topology we are trying to model (aka scenario), then modifying the topology / conceptual model enables greater clarity on what key aspects of the characterization need to change to best represent the alternate scenario. The emphasis is on the effective representation of alternate scenarios in preference to performing parametric sensitivities around a preferred best technical case. 